\title{
Thermodynamic properties of hydrophobic deep eutectic solvents and solubility of water and HMF in them
}

\section{Citation for published version (APA):}

Dietz, C. H. J. T., Erve, A., Kroon, M. C., van Sint Annaland, M., Gallucci, F., \& Held, C. (2019). Thermodynamic properties of hydrophobic deep eutectic solvents and solubility of water and HMF in them: measurements and PC-SAFT modeling. Fluid Phase Equilibria, 489, 75-82. https://doi.org/10.1016/j.fluid.2019.02.010

\section{Document license: \\ TAVERNE}

DOI:

10.1016/j.fluid.2019.02.010

Document status and date:

Published: 15/06/2019

\section{Document Version:}

Publisher's PDF, also known as Version of Record (includes final page, issue and volume numbers)

\section{Please check the document version of this publication:}

- A submitted manuscript is the version of the article upon submission and before peer-review. There can be important differences between the submitted version and the official published version of record. People interested in the research are advised to contact the author for the final version of the publication, or visit the $\mathrm{DOI}$ to the publisher's website.

- The final author version and the galley proof are versions of the publication after peer review.

- The final published version features the final layout of the paper including the volume, issue and page numbers.

Link to publication

\section{General rights}

Copyright and moral rights for the publications made accessible in the public portal are retained by the authors and/or other copyright owners and it is a condition of accessing publications that users recognise and abide by the legal requirements associated with these rights.

- Users may download and print one copy of any publication from the public portal for the purpose of private study or research.

- You may not further distribute the material or use it for any profit-making activity or commercial gain

- You may freely distribute the URL identifying the publication in the public portal.

If the publication is distributed under the terms of Article 25fa of the Dutch Copyright Act, indicated by the "Taverne" license above, please follow below link for the End User Agreement:

www.tue.nl/taverne

Take down policy

If you believe that this document breaches copyright please contact us at:

openaccess@tue.nl

providing details and we will investigate your claim. 


\title{
Thermodynamic properties of hydrophobic deep eutectic solvents and solubility of water and HMF in them: Measurements and PC-SAFT modeling
}

\author{
Carin H.J.T. Dietz ${ }^{\mathrm{a}, \mathrm{b}}$, Annika Erve ${ }^{\mathrm{c}}$, Maaike C. Kroon ${ }^{\mathrm{d}}$, Martin van Sint Annaland ${ }^{\mathrm{b}}$, \\ Fausto Gallucci ${ }^{\mathrm{a}}$, Christoph Held ${ }^{\mathrm{c}, *}$ \\ a Inorganic Membranes and Membrane Reactors, Department of Chemical Engineering and Chemistry, Eindhoven University of Technology, PO Box 513, \\ 5600 MB Eindhoven, Netherlands \\ ${ }^{\mathrm{b}}$ Chemical Process Intensification, Department of Chemical Engineering and Chemistry, Eindhoven University of Technology, PO Box 513,5600 MB \\ Eindhoven, Netherlands \\ ${ }^{c}$ Laboratory of Thermodynamics, Department of Biochemical and Chemical Engineering, TU Dortmund University, Emil-Figge-Str. 70, 44227 Dortmund, \\ Germany \\ d Khalifa University of Science and Technology, Petroleum Institute, Chemical Engineering Department, P.O. Box 2533, Abu Dhabi, United Arab Emirates
}

\section{A R T I C L E I N F O}

\section{Article history:}

Received 27 November 2018

Received in revised form

5 February 2019

Accepted 6 February 2019

Available online 13 February 2019

\section{Keywords:}

DES

Vapor pressure

Density

5-Hydroxymethylfurfural

Green solvents

\begin{abstract}
A B S T R A C T
Recently, hydrophobic deep eutectic solvents (DESs) were proposed as interesting solvents for biorefinery processes, such as the production of 5-hydroxymethylfurfural (HMF) from glucose in an aqueous environment. Physicochemical property data of hydrophobic DES + water/HMF systems are of utmost importance for process design. In this work, density and vapor pressure of eight different hydrophobic DESs, as well as water solubility and HMF solubility in these DESs were experimentally determined. The solubility was modeled using the pseudo-pure component approach within the framework of PerturbedChain Statistical Associating Fluid Theory (PC-SAFT). New pure-component parameters for the eight hydrophobic DESs were obtained by fitting to measured density and vapor-pressure data, instead of to density data only. Based on these new pure-component parameters for the DESs, the solubilities of water and of HMF in the hydrophobic DESs were modeled in good agreement with the experimental data at $298 \mathrm{~K}$ and atmospheric pressure.
\end{abstract}

() 2019 Elsevier B.V. All rights reserved.

\section{Introduction}

In the last decade, deep eutectic solvents (DESs) were proposed as a new class of sustainable designer solvents [1]. DESs are composed of two or more (solid) components, which most likely interact via hydrogen bonding and/or Van der Waals interactions [2,3]. These physical interactions significantly lower the melting temperature of the mixture compared to the melting temperature of its constituents, and stabilize the configuration of the liquid at room temperature. The first DESs reported in literature were composed of combinations of amides and choline chloride [4,5]. Because of the strong hydrogen-bond interactions in these mixtures, these DESs also showed strong interaction with water and

\footnotetext{
* Corresponding author.

E-mail address: christoph.held@tu-dortmund.de (C. Held).
}

were therefore hydrophilic and hygroscopic. As these DESs were water-miscible, they were unsuited as extracting agents for the liquid-liquid extraction of products from aqueous phases. Instead, hydrophobic DESs, which consist of hydrophobic constituents and minimize the water-content in the DESs, can be used for this purpose and they were reported for the first time in literature in 2015 [6].

Even though hydrophobic DESs are relatively new, already several applications have been published, including the separation of caffeine and vanillin [6,7], metal ions [8,9] and pesticides [10] from an aquatic environment by means of liquid-liquid extraction, and the removal of furfural and 5-hydroxymethylfurfural (HMF) from water by the use of DES-impregnated membrane technology [11]. Furthermore, hydrophobic DESs were used for the capture of $\mathrm{CO}_{2}[12,13]$ and applied as micro-extracting agents in emulsions [14]. Finally, hydrophobic DESs have been used for the extraction of components from leaves $[15,16]$. 
For all these applications, it is important that the mutual solubility of hydrophobic DES and water is minimized. Furthermore, it is desirable that the constituents of the DES are sustainable (and environmentally friendly). Few hydrophobic DESs that fulfill both requirements have already been presented in literature [17]. However, it is expected that many more hydrophobic DESs exist, which have yet to be discovered. Reason is that there is an almost infinite choice in selecting both constituents and their ratio. Experiments for DES' design and the selection of the best performing DESs for specific applications are very time-consuming. Thus, it is extremely important to develop robust models that are able to predict the solid-liquid phase diagram of DES constituents and the thermophysical properties of hydrophobic DESs.

The thermodynamic model Perturbed-Chain Statistical Associating Fluid Theory (PC-SAFT) was found to be able to successfully predict several physicochemical properties of hydrophobic DESs $[12,18]$. However, in these works the pure-component parameters of the DES constituents were fitted to density data only. Recently, vapor-pressure data have become available for several hydrophobic DESs [18] and these data will be used in this study in order to obtain improved pure-component parameters that are fitted to both, density data and vapor-pressure data.

In this work, PC-SAFT will be applied to predict the phase behavior of mixtures containing one hydrophobic DES and either water or HMF. Eight hydrophobic DESs were considered in this work. These mixtures are important in biorefinery processes, where DESs can be used as a solvent for the production of HMF from sugars in an aqueous environment [19-21]. Further, it is important to quantify the amount of water that can maximally be present in a liquid DES in contact with aqueous phases. Thus, this work aims at predicting solubility of water in DES phases that are in equilibrium with the aqueous phase, as well as the solubility of furfural and HMF in "water-free" DESs. The predicted solubilities will finally be compared to new experimental data that will be presented in this work as well; this data includes saturated water contents of the DES phase which is in equilibrium with a DES-poor water phase, as well as solid solubility data of HMF in the pure DESs.

\section{Materials and methods}

\subsection{Chemicals}

The chemicals used in this work, including their purity, source and melting point, are presented in Table 1 . All chemicals were used as obtained from the suppliers.

\subsection{DESs preparation}

Table 2 lists the eight different hydrophobic DESs prepared in this work, including their hydrogen bond donors (HBDs), hydrogen bond acceptors (HBAs) and the molar ratio between HBD and HBA. Both room-temperature solids (HBD and HBA) were weighed using a Mettler Ax205 balance with an accuracy of $\pm 0.2 \cdot 10^{-4} \mathrm{~g}$ and mixed in the desired molar ratio in a round bottom flask, to produce an approximate amount of $25 \mathrm{~g}$ of each DES. The round bottom flask was stirred and heated in an oil bath at $313 \mathrm{~K}$ for $2 \mathrm{~h}$ to obtain a liquid, the DES. An IKA RCT basic magnetic stirrer was used for monitoring the stirring speed and temperature. An IKA ETS-D5 temperature controller with an uncertainty of $\pm 0.1 \mathrm{~K}$ was used for temperature control. The DES remained liquid after cooling to room temperature. To ensure the validity of the measurements, each DES was prepared at least in two independent syntheses.

\subsection{Density of DESS}

The density of the hydrophobic DESs was measured with an Anton-Paar DMA $4500 \mathrm{M}$ vibrating tube with a density accuracy of $\pm 50 \cdot 10^{-6} \mathrm{~g} \mathrm{~cm}^{-3}$ and a temperature accuracy of $\pm 0.05 \mathrm{~K}$. The discrepancy of the density from several reference oils that were measured was not higher than $\pm 0.00001 \mathrm{~g} \mathrm{~cm}^{-3}$.

\subsection{Total vapor pressure of the DESs}

\subsubsection{Experimental method}

Vapor pressures of the prepared hydrophobic DESs were measured using a HS20 head space, a GC-2010-plus gas chromatograph of Shimadzu (with a capillary 100\% dimethylpolysiloxane Agilent DB-1MS ultra inert column with a length of $30 \mathrm{~m}$, diameter of $0.25 \mathrm{~mm}$ and a film thickness of $0.25 \mu \mathrm{m}$ ), and a MS QP2020 of Shimadzu. The head-space keeps the sample at a certain temperature, with an accuracy of $\pm 0.5 \mathrm{~K}$, for a specified period of time. By means of GC and MS, the constituents were separated and detected [22,23]. The sample line temperature has an accuracy $\pm 0.5 \mathrm{~K}$ and the transfer line temperature $\pm 0.5 \mathrm{~K}$. The GC-2010-plus has an accuracy in temperature $\pm 1 \%$ (calibration at $0.01 \mathrm{~K}$ ). Helium was used as carrier gas and the settings of the HS-GC-MS can be found in Table $S 1$ in the supporting information. $0.5 \mathrm{~g}$ DES was put in $20 \mathrm{ml}$ vials and incubated for different times (5-15-30-60$120 \mathrm{~min}$ ) at different temperatures (313-333-353-373 K). After incubation, $1 \mathrm{~mL}$ of the gas-phase was sampled and the concentrations of the DESs constituents in the gas-phase were analyzed with GC-MS. From these concentrations vapor-pressure data were obtained using the Clausius-Clapeyron equation. Vapor-pressure measurements were repeated five times, and the standard deviation was found to vary between 3.4 and $20 \mathrm{~Pa}$, depending on the DES structure and temperature.

\subsubsection{Vapor-pressure modeling}

The $\varphi-\varphi$ approach was used in this work to model the vaporliquid equilibrium of the DESs under investigation at various temperatures yielding the total vapor pressure at constant

Table 1

Chemicals used, including purity, melting point $\left(T_{m}\right)$ (both provided by the supplier), provider and CAS number.

\begin{tabular}{|c|c|c|c|c|}
\hline Name & Purity & Provider & CAS number & $T_{\mathrm{m}}(\mathrm{K})$ \\
\hline Decanoic acid & $>98 w t \%$ & Sigma Aldrich & $334-48-5$ & $300-305$ \\
\hline Dodecanoic acid & $>98 w t \%$ & Sigma Aldrich & $143-07-7$ & $316-318$ \\
\hline Furfural & $>99 \mathrm{wt} \%$ & Sigma Aldrich & $98-01-1$ & 237 \\
\hline 5-Hydroxymethylfurfural & $\geq 99 \mathrm{wt} \%$ & Sigma Aldrich & $67-47-0$ & $301-307$ \\
\hline$n$-Tetraoctylammonium bromide & -98 wt\% & Sigma Aldrich & $14866-33-2$ & $368-371$ \\
\hline Thymol & $>99 w t \%$ & TCI Chemicals & $89-83-8$ & $322-325$ \\
\hline Menthol & $>99 w t \%$ & Sigma Aldrich & $98-78-1$ & 304 \\
\hline Lidocaine & $>99 w t \%$ & Sigma Aldrich & $137-58-6$ & $339-342$ \\
\hline HYDRANAL ${ }^{\mathrm{TM}}$-Chloroform & - & HONEYWELL Fluka ${ }^{\mathrm{TM}}$ & $67-66-3$ & - \\
\hline HYDRANAL $^{\mathrm{TM}}$-Composit 2 & - & HONEYWELL FlukaTM & - & - \\
\hline
\end{tabular}


Table 2

DESs prepared in this work including their HBD, HBA, HBD:HBA ratio and abbreviation.

\begin{tabular}{|c|c|c|c|}
\hline HBD & HBA & Molar ratio HBD:HBA & Abbreviation \\
\hline Decanoic acid & $n$-Tetraoctylammonium bromide & $2: 1$ & deca-n8888Br \\
\hline Decanoic acid & Thymol & $1: 1$ & deca-thy \\
\hline Decanoic acid & Lidocaine & $2: 1$ & deca-lid 2:1 \\
\hline Decanoic acid & Lidocaine & $3: 1$ & deca-lid 3:1 \\
\hline Decanoic acid & Lidocaine & $4: 1$ & deca-lid $4: 1$ \\
\hline Decanoic acid & Menthol & $1: 1$ & deca-men \\
\hline Thymol & Lidocaine & $2: 1$ & thy-lid \\
\hline Dodecanoic acid & Lidocaine & $2: 1$ & dode-lid \\
\hline
\end{tabular}

composition. The DESs were considered as a pseudo-pure system, i.e. the pseudo-pure component modeling approach was applied in this work. Thus, the approach does not differ from fitting parameters for conventional organic solvents to according experimental data, e.g. Ref. [26].

\subsection{Water content of the DES phase}

The liquid-liquid equilibrium of systems water + hydrophobic DES was not characterized completely in this work. That is, only the water solubility in the DES-rich phase (after contacting and equilibration with water) was measured rather than the complete mutual solubilities of water and DES. Two different methods were applied in this work, which are presented in the following. The water content of the DESs before mixing with water was also determined and are reported in supporting information Table S2.

\subsubsection{Equilibration method}

Around $0.5 \mathrm{~g}$ of a DES was placed in a $1.5 \mathrm{ml}$ Eppendorf ${ }^{\mathbb{R}}$ tube, and about $0.5 \mathrm{~g}$ of water was added by means of a disposable $2 \mathrm{ml}$ syringe. Each step was accompanied by weighing using the balance XS205 DualRange by Mettler Toledo (Wien, Austria). The sample was then placed in a Thermomixer C from Eppendorf (WesselingBerzdorf, Germany) and it was shaken at $298 \mathrm{~K}$ and $1100 \mathrm{rpm}$ for about $2 \mathrm{~h}$. Subsequently, the sample was centrifuged in the bench centrifuge $5418 \mathrm{R}$ from Eppendorf (Wesseling-Berzdorf, Germany) at $298 \mathrm{~K}$ and 14,000 rpm for $15 \mathrm{~min}$. Finally, the sample was allowed to settle for at least $2 \mathrm{~h}$.

The centrifugation step was not required for the mixtures with the DESs deca-men, deca-thy or thy-lid; this was validated by analyzing and comparing the LLE data with and without centrifugation step, which yielded same results within experimental uncertainty. However, the centrifugation step was crucially required for mixtures containing the DES deca-lid (each molar ratio) or decan $8888 \mathrm{Br}$. In these systems, three apparent phases could be observed if too much water was added at once, and local inhomogeneity could only be removed by centrifugation.

\subsubsection{Optical method}

The results of the above-described method were additionally validated with an optical method. Around $0.5 \mathrm{ml}$ of a DES was placed in a $1.5 \mathrm{ml}$ Eppendorf ${ }^{\mathrm{B}}$ tube and weighed. Then a drop of water was added by means of a disposable syringe. The sample was then placed in a Thermomixer $C$ from Eppendorf (WesselingBerzdorf, Germany). The temperature was set to $298 \mathrm{~K}$ and the shaking frequency to $1100 \mathrm{rpm}$. After a settling time of $20 \mathrm{~min}$ it was visually checked whether the system was phase-separated or not. This process was repeated until a second phase was detected, and the amount of added water was recorded by differential weighing. This method has a very high uncertainty, and it was only applied for the purpose of cross-validation.

\subsubsection{Analysis}

In order to analyze the water content in the DES phase, samples were drawn carefully from the DES phase, which was the upper phase for all systems, and analyzed by Karl-Fischer-titration. The automatic titrator $915 \mathrm{KF}$ Ti-Touch from Metrohm (Herisau, Switzerland) was used. The measurement is a volumetric determination with a one-component reagent. To avoid esterification of the carbonic acid (which is a constituent of all the considered DESs) within the DES by methanol, the chloroform-based reagent HYDRANALTM chloroform from Honeywell Fluka ${ }^{\mathrm{TM}}$ (Bucharest, Romania) was used. The titrant used was HYDRANAL Composite 2 from Honeywell Fluka ${ }^{\mathrm{TM}}$. In a first step, the titer was determined. For this, a droplet of pure water was added to chloroform through the septum using a disposable syringe. This procedure was repeated three times and the titer was averaged. After determining the titer, the samples were analyzed and the water content of the DES phase was calculated by means of the titer and the differential weighing using the balance TE $214 \mathrm{~S}$ from the company Sartorius (Göttingen, Germany), which transmitted the mass of the injected sample. The analysis of the sample was carried out four times in this work, and the values were averaged.

\subsubsection{LLE modeling}

The water content of the hydrophobic DES phase (DES) equilibrated with a water-rich phase (aq) that contains low amounts of DES phase was modeled using the iso-activity criterion, which can be applied for low absolute pressures:

$x_{\text {water }}^{(a q)} \gamma_{\text {water }}^{(a q)}=x_{\text {water }}^{(D E S)} \gamma_{\text {water }}^{(D E S)}$

The required activity coefficients of water $\gamma_{\text {water }}$ in both phases were predicted with PC-SAFT in order to finally obtain the solubility of water in the DES phase, expressed as mole fraction of water $x_{\text {water }}$ in the DES phase.

\subsection{Solubility of $H M F$}

\subsubsection{Solubility measurements}

The solubilities of furfural and of HMF were measured in three selected hydrophobic DESs (in duplo) by the cloud-point method. Five grams of every DES were weighed in a vial. The vials were heated to one of the desired temperatures ( $303 \mathrm{~K}$ ). Every $2 \mathrm{~h}$ a small amount of HMF was added. Only if the small amount was dissolved after $2 \mathrm{~h}$, the experiment was continued by adding again a weighed amount of HMF. This was repeated until full saturation was achieved. The measurements in duplo yielded standard uncertainties $\mathrm{u}(\mathrm{w})=0.005, \mathrm{u}(\mathrm{T})=0.2 \mathrm{~K}$.

\subsubsection{Solubility modeling}

Solubility of HMF in the hydrophobic DESs were modeled in this work using a simplified equilibrium condition according to Prausnitz [24] and Gmehling et al. [25]. 
$\ln x_{H M F}^{l} \gamma_{H M F}^{l}=-\frac{\Delta h_{H M F}^{f u s}}{R T}\left(1-\frac{T}{T_{H M F}^{m}}\right)$

This equation assumes a pure solid HMF phase (no mixed waterHMF crystals) and further neglects the temperature dependency of the heat capacities. $\Delta h_{H M F}^{f u s}$ and $T_{H M F}^{m}$ represent the molar fusion enthalpy and the temperature of melting of pure HMF, respectively. The influence of the medium on the solubility of HMF is described by the activity coefficient $\left(\gamma_{H M F}^{l}\right)$, which mimics the molecular selfinteractions and cross-interactions in the liquid phase. The melting properties for HMF were taken from literature [31].

\subsection{PC-SAFT}

It can be observed from equations (1) and (2) that activity coefficients are required for predicting the water solubility in a DES phase and the HMF solubility in a pure DES, respectively. In this work, activity coefficients were predicted with PC-SAFT [26], which calculates the residual Helmholtz energy as the sum of the energy contributions due to hard-chain repulsion $a^{h c}$, dispersion $a^{\text {disp }}$, and association $a^{a s s o c}$ finally yielding the residual Helmholtz energy $a^{\text {res : }}$

$a^{r e s}=a^{h c}+a^{d i s p}+a^{a s s o c}$

Once $a^{\text {res }}$ is computed from equation (3) thermodynamic properties (e.g., fugacities) can be derived from the volume and composition dependency of $a^{\text {res }}$, which is textbook thermodynamics (e.g. Refs. [26,28]). To describe mixtures, combining and mixing rules were applied, namely the conventional BerthelotLorentz rules including one additional binary parameter $k_{i j}$ that corrects the dispersion-energy parameter for the mixture $u_{i j}$ from the geometric mean of the self-dispersion energy parameters $u_{i}$ and $u_{j}$

$u_{i j}=\sqrt{u_{i} u_{j}}\left(1-k_{i j}\right)$

The cross-association energy between two associating substances $i$ and $j$ was characterized using the rules from Wolbach and Sandler [27].

$\varepsilon^{A_{i} B_{j}}=\frac{1}{2}\left(\varepsilon^{A_{i} B_{i}}+\varepsilon^{A_{j} B_{j}}\right)$

$\kappa^{A_{i} B_{j}}=\sqrt{\kappa^{A_{i} B_{i}} \kappa^{A_{j} B_{j}}}\left(\frac{\sqrt{\sigma_{i i} \sigma_{j j}}}{\frac{1}{2}\left(\sigma_{i i}+\sigma_{j j}\right)}\right)^{3}$

with the Boltzmann constant $k_{B}$, the association-energy parameter $\varepsilon^{A_{i} B_{i}} / k_{B}$, and the association-volume parameter $\kappa^{A_{i} B_{i}}$, as well as the segment diameter $\sigma_{i}$

\section{Results}

\subsection{Experimental results}

\subsubsection{Density data of pure hydrophobic DESs}

The density of the liquid DESs between $293 \mathrm{~K}$ and $328 \mathrm{~K}$ at $101 \mathrm{kPa}$ are presented in Fig. 1, and the experimental data is listed in Table S3 in the Supporting information.

As can be observed from Fig. 1, the liquid density decreases linearly with temperature, and the slope of the lines is very similar for the different DESs under consideration. The density of the hydrophobic DESs are in the same order of magnitude as other DESs [12].

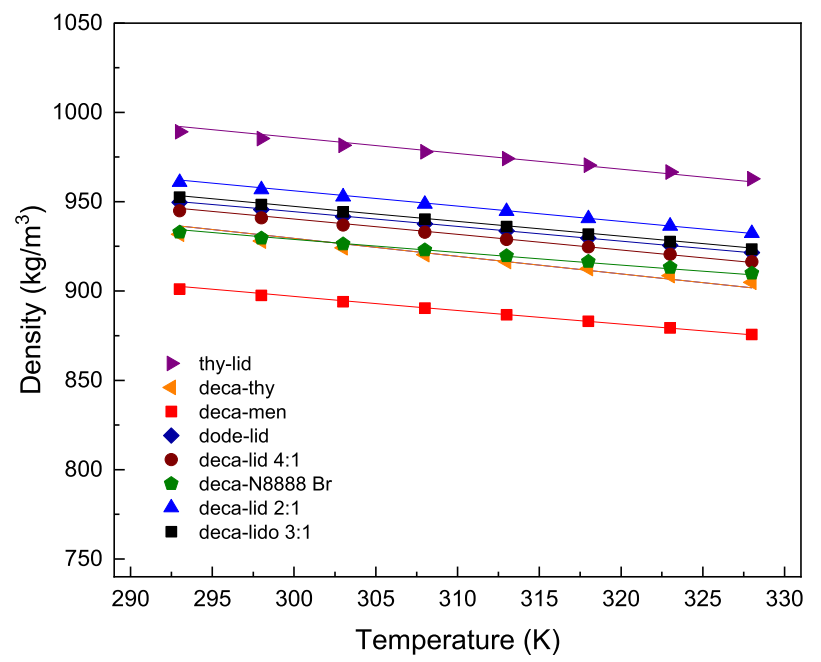

Fig. 1. Densities of deca-lid 2:1, deca:lid 3:1, deca:lid 4:1, dode-lid, deca:men, deca:thy and thy:lid as a function of the temperature at $\mathrm{p}=101 \mathrm{kPa}$. The symbols represent the experimental data and the solid lines represent the PC-SAFT results using the parameters from Table 7 .

\subsubsection{Total vapor pressures of pure hydrophobic DESs}

The vapor pressures were measured for the DES deca-n $8888 \mathrm{Br}$. For all other DESs, the vapor-pressure data was already available from literature [18]. The results are listed in Table 3. As expected, the volatility of deca-n $8888 \mathrm{Br}$ is very low, because of the low vapor pressure of n8888Br, which also influences the vapor pressure of deca and the total vapor pressure. The total vapor pressure of deca$\mathrm{n} 8888 \mathrm{Br}$ is in the same order of magnitude as the hydrophobic DESs [18]. The same holds true for the data of dode-lid.

\subsubsection{Experimental HMF solubility data in hydrophobic DESs}

The solubility of HMF in the liquid DESs at $303 \mathrm{~K}$ and $101 \mathrm{kPa}$ are presented in Table 4. Exemplarily, only HMF solubility in three different DESs was measured, namely in deca-thy, deca-n8888Br and thy-lid, respectively. Considering the data presented in Table 3, it becomes obvious that the solubility of HMF in all three DESs is very high; the solubility varies between $75 \mathrm{w} \%$ and $85 \mathrm{w} \%$. Although not shown, miscibility data were also recorded at higher temperatures ( $323 \mathrm{~K}$ and $373 \mathrm{~K}$ ), and as a result it could be observed that the miscibility of HMF/DES mixtures does hardly depend on temperature within the considered temperature range and in the DESs under investigation.

\subsubsection{Experimental water solubility data in hydrophobic DES phase}

Two different methods were applied to measure the liquidliquid equilibrium, an optical method upon stepwise adding water to a homogeneous DES phase, and an equilibration method that combines mixing, settling and centrifugation. The latter is the preferred method. The results using this method are listed in

Table 3

Partial pressures of the constituents of the DESs dode-lid and deca-n $8888 \mathrm{Br}$ and their total vapor pressures at different temperatures measured in this work.

\begin{tabular}{llll}
\hline$T[\mathrm{~K}]$ & $p_{\text {dodecanoic acid }}[\mathrm{Pa}]$ & $p_{\text {lidocaine }}[\mathrm{Pa}]$ & $p^{\text {tot }}[\mathrm{Pa}]$ \\
\hline 333 & 3 & 0 & 3 \\
353 & 12 & 1 & 13 \\
373 & 34 & 2 & 36 \\
$T[\mathrm{~K}]$ & $p_{\text {decanoic acid }}[\mathrm{Pa}]$ & $p_{\text {n8888Br }}[\mathrm{Pa}]$ & $p^{\text {tot }}[\mathrm{Pa}]$ \\
353 & 11 & 0 & 11 \\
373 & 39 & 0 & 39 \\
\hline
\end{tabular}

Standard uncertainties are $u(T)=0.5 \mathrm{~K}$ and $u(p)=10 \%$ 
Table 4

Solubility of HMF in three selected DESs at $T=303 \mathrm{~K}$ and $p=101 \mathrm{kPa}$.

\begin{tabular}{|c|c|c|c|c|}
\hline Solubility & & deca-thy & deca-n8888Br & thy-lid \\
\hline $\mathrm{w}_{\mathrm{HMF}}$ & [\%] & 80 & 75 & 85 \\
\hline
\end{tabular}

Standard uncertainties are $u(w)=0.005, u(T)=0.2 \mathrm{~K}$ and $u(p)=3 \mathrm{kPa}$.

Table 5

Experimental water weight fraction in different DESs at $T=298 \mathrm{~K}$ and $p=101 \mathrm{kPa}$. P1, P2 and P3 denote three independent syntheses of each of the DESs. Data obtained with the analytical equilibrium method.

\begin{tabular}{llll}
\hline DES & $\boldsymbol{w}_{\mathbf{H 2 O}}^{\text {DES }}$ P1 [wt. \%] & $\boldsymbol{w}_{\mathbf{H 2 O}}^{\text {DES }}$ P2 [wt. \%] & $\boldsymbol{w}_{\mathbf{H 2 O}}^{\text {DES P3 [wt. \%] }}$ \\
\hline deca-lid 4:1 & 6.34 & 6.27 & - \\
deca-lid 3:1 & 7.79 & 8.70 & - \\
deca-lid 2:1 & 14.43 & 17.70 & 15.46 \\
deca-men 1:1 & 2.16 & - & - \\
deca-thy 1:1 & 3.42 & - & - \\
deca-n8888Br 2:1 & 3.62 & 2.49 & 2.24 \\
thy-lid 2:1 & 1.72 & - & - \\
\hline
\end{tabular}

Standard uncertainties are $u(T)=0.2 \mathrm{~K}$ and $u(p)=3 \mathrm{kPa}$ and $u_{\text {deca-lid }{ }_{4: 1}\left(w_{\text {water }}^{\text {DES }}\right.}$ $\mathrm{P} 1)=0.15 \mathrm{wt} \%, u_{\text {deca-lid } 4: 1}\left(w_{\text {water }}^{\text {DES }} \mathrm{P} 2\right)=0.13 \mathrm{wt} \%, u_{\text {deca-lid } 3: 1}\left(w_{\text {water }}^{\text {DES }} \mathrm{P} 1\right)=0.14 \mathrm{wt} \%$, $u_{\text {deca-lid } 3: 1}\left(w_{\text {water }}^{\text {DES }} \quad \mathrm{P} 2\right)=0.51 \mathrm{wt} \quad \%, \quad u_{\text {deca-lid } 2: 1}\left(w_{\text {water }}^{D E S} \mathrm{P} 1\right)=0.15 \mathrm{wt} \quad \%, \quad u_{\text {deca- }}$ lid 2:1 $\left(w_{\text {water }}^{\text {DES }} \mathrm{P} 2\right)=0.51 \mathrm{wt} \quad \%, \quad u_{\text {deca-lid } 2: 1}\left(w_{\text {water }}^{\text {DES }} \mathrm{P}\right)=0.32 \mathrm{wt} \quad \%, \quad u_{\text {deca }}$ men 1:1 $\left(w_{\text {water }}^{D E S} \mathrm{P} 1\right)=0.16 \mathrm{wt} \quad \%, \quad u_{\text {deca-thy } 1: 1}\left(w_{\text {water }}^{D E S} \mathrm{P} 1\right)=0.10 \mathrm{wt} \quad \%, \quad u_{\text {deca }}$ $n 8888 B \mathrm{Br} 2: 1\left(w_{\text {water }}^{D E S} \mathrm{P} 1\right)=0.05 \mathrm{wt} \%, \quad u_{\text {deca-n8888Br 2:1 }}\left(w_{\text {water }}^{\mathrm{DES}} \mathrm{P} 2\right)=0.40 \mathrm{wt} \%, \quad u_{\text {deca }}$ $n 8888 B$ r 2:1 $\left(w_{\text {water }}^{\text {DES }} \mathrm{P} 3\right)=0.07$ wt \%, $u_{\text {thy-lid } 2: 1}\left(w_{\text {water }}^{\text {DES }} \mathrm{P} 1\right)=0.10$ wt $\%$

Table 5, which show that the LLE measurements of DES + water mixtures using three independently synthesized DESs agree well with each other (samples P1-P2-P3 in one line). Further, some conclusions can be drawn from these results: In general, the water content of DESs is about $2 \mathrm{wt} \%$, except for those of the combination of decanoic acid with lidocaine. The combination of deca-lid causes much higher water contents in the DES phase than for mixtures with other DESs. The fact that especially the DESs containing big amounts of lidocaine contain the comparatively highest amounts of water is surprising as lidocaine itself is only marginally soluble in water.

The results obtained with the equilibration method were further compared to measurements with the optical method. The latter are presented in Table 6 . It can be nicely seen that the results obtained with both methods are in very good agreement. It should be stressed that the optical method only yields reasonable results, if clearly two separated liquid phases are observed upon addition of water to DES. Note, that for other systems (e.g. water + deca-lid) additional local phases were observed. In these cases, phase equilibrium was not reached completely and the equilibration method (shaking + settling + centrifugation) is required.

\section{Table 6}

Experimental water weight fractions in the DES phase of different water + DES mixtures at $T=298 \mathrm{~K}$ and $p=101 \mathrm{kPa} . \mathrm{KF}_{\mathrm{opt}}$ and $\mathrm{KF}_{\mathrm{eq}}$ mean that all the phases were analyzed by Karl-Fischer-titration; however, "opt" denotes the optical observation of the liquid-liquid demixing, while "eq" means the equilibration of the two liquid phases by shaking + settling + centrifugation.

\begin{tabular}{lll}
\hline DES & Method & $\boldsymbol{w}_{\mathbf{H} 2 \boldsymbol{O}}^{\text {DES }}[\mathrm{wt} . \%]$ \\
\hline deca-men $1: 1$ & $\mathrm{KF}_{\text {opt }}$ & 2.17 \\
& $\mathrm{KF}_{\text {eq }}$ & 2.16 \\
deca-thy $1: 1$ & $\mathrm{KF}_{\text {opt }}$ & 3.43 \\
\multirow{2}{*}{ thy-lid 2:1 } & $\mathrm{KF}_{\text {eq }}$ & 3.42 \\
& $\mathrm{KF}_{\mathrm{opt}}$ & 1.57 \\
& $\mathrm{KF}_{\text {eq }}$ & 1.72 \\
\hline
\end{tabular}

Standard uncertainties are $u(T)=0.2 \mathrm{~K}$ and $u(p)=3 \mathrm{kPa}$ and $u_{\text {deca-men } 1: 1}\left(w_{\text {water }}^{D E S}\right.$ $\left.K F_{\text {opt }}\right)=0.06 \mathrm{wt} \%, \quad u_{\text {deca-men 1:1 }}\left(w_{\text {water }}^{\text {DES }} \quad K F_{\text {eq }}\right)=0.16 \mathrm{wt} \%, \quad u_{\text {deca-thy } 1: 1}\left(w_{\text {water }}^{\text {DES }}\right.$ $\left.K F_{\text {opt }}\right)=0.10 \mathrm{wt} \%, \quad u_{\text {deca-thy 1:1 }}\left(w_{\text {water }}^{D E S} \quad K F_{\text {eq }}\right)=0.10 \mathrm{wt} \%, \quad u_{\text {thy-lid 2:1 }}\left(w_{\text {water }}^{\text {DES }}\right.$ $\left.K F_{\text {opt }}\right)=0.02 \mathrm{wt} \%, u_{\text {thy-lid 2:1 }}\left(w_{\text {water }}^{D E S} K F_{\text {eq }}\right)=0.09$ wt \%.

\subsection{Estimated pure-component PC-SAFT parameters for the hydrophobic DESs}

Two main modeling approaches have been established for characterizing DES in the PC-SAFT framework [28]. The first is an individual-component modeling approach in which both of the DES constituents are explicitly accounted for in the modeling. The second approach neglects the fact that DESs are composed of two different constituents, and rather one DES is treated as a pseudopure component. In this work, the second approach was followed, i.e. the DESs were considered as pseudo-pure components. Modeling phase equilibria with PC-SAFT requires estimating the five pure-component parameters $m_{s e g, i}, \sigma_{i}$, and $u_{i} / k_{B}, \varepsilon^{A i B i} / k_{B}$ and $k^{A i B i}$. In addition, the number of association sites $N^{s i t e}$ (association scheme) must be set prior to calculations. The latter was not treated as a fitting parameter. Rather, for the hydrophobic DESs, a $2 \mathrm{~B}$ association scheme was applied according to previous works on PCSAFT modeling DESs and DES-based mixtures [12-28]. In previous works, the pure-component parameters were fitted to liquid density data of the DESs. In this work, a new strategy was followed, as a previous work provided total vapor pressures of the DESs. The pure-component parameters, $m_{\text {seg, } i,}, \sigma_{i}$, and $u_{i} / k_{B}, \varepsilon^{A i B i} / k_{B}$ and $k^{A i B i}$ of the designated DESs were fitted to liquid density data and vaporpressure data from literature and from this work using the objective function $\mathrm{OF}$, which weighs all data points equally.

$O F=\sum_{i}^{N P \text { density }}\left(\frac{\rho^{\text {mod }}-\rho^{\text {exp }}}{\rho^{\exp }}\right)^{2}+\sum_{i}^{N P \text { vapor pressure }}\left(\frac{p^{\text {mod }}-p^{\text {exp }}}{p^{\exp }}\right)^{2}$

It should be noted that in previous works on modeling DESs the association parameters $\varepsilon^{A i B i} / k_{B}$ and $k^{A i B i}$ were set to constant values [12-28]. This was not possible in this work for a fair description of vapor-pressure data. Thus, $\varepsilon^{A i B i} / k_{B}$ and $k^{A i B i}$ were also treated as adjustable parameters. Further, it should be mentioned that the applied pseudo-pure component modeling approach requires a new PC-SAFT pure-component parameter set for each new composition of one DES, which might be considered as drawback of the suggested approach. However, in many cases only DESs at certain compositions are used in certain applications, and in this case the pseudo-pure component modeling approach combines simplicity with speed advantages, especially if the approach will be applied in process design tools for multi-component systems.

Applying the $O F$ and the described modeling strategy within the PC-SAFT framework yielded the pure-component parameters listed in Table 7. Although the DESs considered here consist of two components at fixed composition, PC-SAFT pseudo-pure component parameters are in reasonable ranges. The parameters for the DES deca-n $8888 \mathrm{Br}$ were available from literature. These parameters were adjusted solely to density data, and the association parameters were set to constant values. In contrast, these parameters were adjusted in this work also to vapor-pressure and density data. As a result, the association-energy parameters range between $1500 \mathrm{~K}$ and $4000 \mathrm{~K}$, respectively, which is very convenient. The association-volume parameters are very low for some DESs. It can be observed that for these DESs the association-energy parameters are then comparably high, which is meaningful as both parameters 
Table 7

Pure-component PC-SAFT parameters for DESs, water, and HMF. All components were modeled using the 2B association scheme (one donator and one acceptor site).

\begin{tabular}{|c|c|c|c|c|c|c|c|c|}
\hline Compounds & $M_{\mathrm{w}} / \mathrm{g} \cdot \mathrm{mol}^{-1}$ & $m_{\text {seg,i }}$ & $\sigma_{\mathrm{i}} / \breve{A}$ & $u_{\mathrm{i}} / \mathrm{k}_{\mathrm{B}}(\mathrm{K})$ & $N_{\text {site }}$ & $\varepsilon^{\mathrm{AiBi}} / k_{\mathrm{B}}(\mathrm{K})$ & $\mathrm{k}^{\mathrm{AiBi}}$ & REF. \\
\hline doca-lid 2:1 & 211.66 & 3.049 & 4.813 & 443.68 & 2B & 1548 & 0.00063 & This work \\
\hline deca-lid 4:1 & 247.17 & 5.588 & 4.072 & 273.16 & $2 \mathrm{~B}$ & 3952 & 0.00006 & This work \\
\hline deca-lid 3:1 & 201.54 & 6.479 & 3.591 & 247.80 & 2B & 2818 & 0.00703 & This work \\
\hline deca-lid 2:1 & 192.95 & 6.802 & 3.473 & 249.44 & $2 \mathrm{~B}$ & 2423 & 0.01275 & This work \\
\hline deca-men & 164.27 & 4.897 & 3.721 & 229.72 & $2 \mathrm{~B}$ & 2682 & 0.09560 & This work \\
\hline deca-thy & 161.24 & 3.756 & 4.071 & 332.09 & $2 \mathrm{~B}$ & 3909 & 0.00004 & This work \\
\hline deca-n8888Br & 297.10 & 15.482 & 3.158 & 317.42 & $2 \mathrm{~B}$ & 5000 & 0.10000 & [22] \\
\hline thy-lid & 178.26 & 6.214 & 3.419 & 222.82 & $2 \mathrm{~B}$ & 2409 & 0.09550 & This work \\
\hline water & 18.02 & 1.205 & 2.793 & 353.95 & $2 \mathrm{~B}$ & 2426 & 0.04500 & [29] \\
\hline HMF & 126.44 & 2.310 & 4.038 & 320.38 & $2 \mathrm{~B}$ & 3168 & 0.00100 & [30] \\
\hline
\end{tabular}

are correlated. For these DESs (deca-thy, deca-lid 4:1, deca-lid 3:1) a more balanced ratio between $\varepsilon^{A i B i} / k_{B}$ and $k^{A i B i}$ was used in order to avoid very low numbers of $k^{A i B i}$. However, higher values for $k^{A i B i}$ for these DESs caused much higher deviations between PC-SAFT and experimental vapor-pressure data. Further, also different association schemes were used in this work in order to avoid low values for $k^{A i B i}$. Moreover, parameter estimation only to vapor-pressure data also could not avoid low values for $k^{A i B i}$. Thus, all these different strategies were not successful, i.e. low values for $k^{A i B i}$ were absolutely required for accurately modeling of the vapor pressure data, and the $2 \mathrm{~B}$ association approach yielded the best results. Thus, the $2 \mathrm{~B}$ association approach and allowing also very low values for $k^{A i B i}$ was considered to give the best $O F$ results, and the parameters of this procedure are listed in Table 7.

The results of minimizing the $O F$ are shown in Fig. 1 (liquid density) and Fig. 2 (total vapor pressures). Both figures show an overall good agreement between experimental data and PC-SAFT. In Fig. 1 it can be observed that PC-SAFT slightly overestimates the experimental density at low temperatures and slightly underestimates the liquid density at higher temperatures. Nevertheless, this deviation is very small and typical (or better) then other works (e.g. Ref. [12]).

The absolute average relative deviation, $\operatorname{AARD}(\%)$, between experimental data and PC-SAFT modeling are listed in Table 8. The $\mathrm{AARD}(\%)$ were calculated according to:

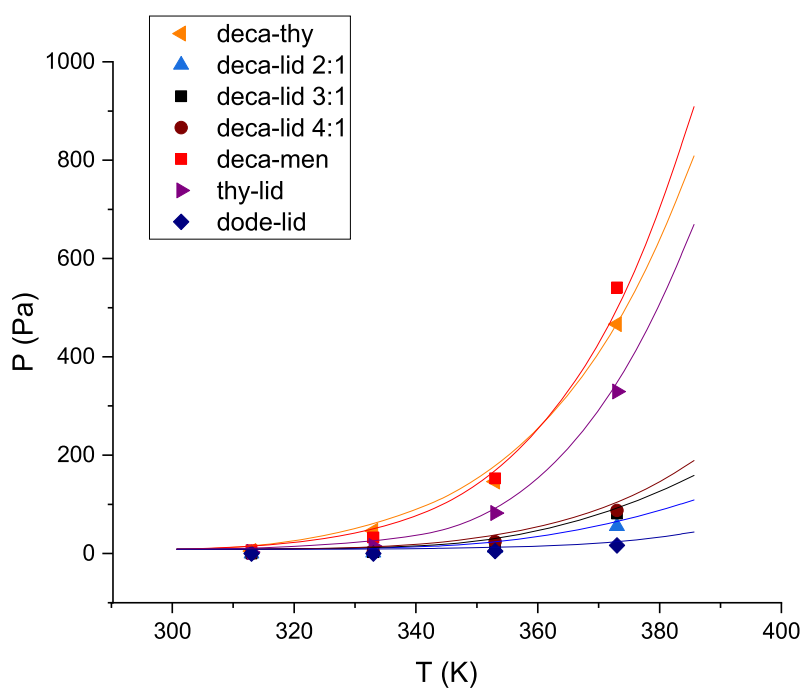

Fig. 2. Total vapor pressures of dode-lid, deca-lid 2:1, deca:lid 3:1, deca:lid 4:1, deca:men, deca:thy and thy:lid, as a function of the temperature. The symbols represent the experimental data from literature [18] and from this work (dode-lid), and the solid lines represent the PC-SAFT results using the parameters from Table 7.
Table 8

Absolute average relative deviation, AARD(\%), between experimental densities and PC-SAFT modeling pure DESs within the temperature range of $288.15-323.15 \mathrm{~K}$ and between experimental vapor pressures and PC-SAFT modeling pure DESs within the temperature range of $285.15-383.15 \mathrm{~K}$.

\begin{tabular}{lllll}
\hline & Density & \multicolumn{3}{l}{ Vapor pressure } \\
\hline DES & No. of data points & AARD (\%) & No. of data points & AARD (\%) \\
doca-lid 2:1 & 8 [this work] & 0.416 & 3 & 4.32 \\
deca-lid 4:1 & 8 [this work] & 0.053 & 5 & 26.49 \\
deca-lid 3:1 & 8 [this work] & 0.012 & 5 & 21.83 \\
deca-lid 2:1 & 8 [this work] & 0.041 & 5 & 17.89 \\
deca-men & 8 [this work] & 0.047 & 5 & 4.95 \\
deca-thy & 8 [this work] & 0.237 & 5 & 12.03 \\
deca-n8888Br & 8 [22] & 0.103 & - & - \\
thy-lid & 8 [this work] & 0.137 & 5 & 3.93 \\
\hline
\end{tabular}

$A A R D(\%)=\frac{1}{n} \sum\left|\frac{y_{i}^{\text {exp }}-y_{i}^{\text {calc }}}{y_{i}^{\text {calc }}}\right| \cdot 100$

In equation (8) $y$ denotes any property determined via experiments (exp) and modeling (calc) for the $n$ number of experimental data points.

The AARD values are not higher than $0.237 \%$ for the density, which means a good agreement between the densities determined via experiments and PC-SAFT. For the density of the DESs deca-lid and deca-men extremely low AARD values were obtained. For the vapor pressures the AARD\% is higher; the maximally obtained value for AARD is $26.49 \%$, which was observed for deca-lid $4: 1$. This comparably higher deviation is probably caused by the strange vapor-pressure behavior, which was discussed in previous work ${ }^{[18]}$.

\subsection{PC-SAFT modeling of water content and the HMF solubility in hydrophobic DESs}

Based on the new PC-SAFT parameters for the DESs under investigation, LLE of water + DES mixtures and SLE of HMF + DES mixtures were predicted. PC-SAFT adequately predicts the water content of the DES phase of the binary water + DES mixtures for the systems containing deca-men, deca-thy, deca-n8888Br and thy-lid. This is shown in Table 9 and in Fig. 3, respectively. PC-SAFT predictions yield water contents of about $3 \mathrm{wt} \%$ for the DES phase of all these mixtures. Further, PC-SAFT also correctly predicts that more water is present in DES phase of systems with thymol than with menthol. Unexpectedly, also the system water + deca-n $8888 \mathrm{Br}$ was predicted in good agreement with the experimental data, despite the fact that the pure-component parameters of this DES were adjusted solely to density data; thus, refitting parameters was avoided. This might point to the fact that mainly steric effects are responsible for the miscibility gap between water and the hydrophobic DESs, which might be easy to predict with PC-SAFT. In 
Table 9

Experimental water weight fractions in the DESs and PC-SAFT predictions at $T=298 \mathrm{~K}$ and $p=101 \mathrm{kPa}$. The experimental data are the averaged values of the samples P1, P2, P3 from Table 5.

\begin{tabular}{|c|c|c|}
\hline DES & $\boldsymbol{w}_{\mathbf{H} 2 \mathrm{O}}^{\boldsymbol{D E S}}[\mathrm{wt} . \%]$ experimental & $\boldsymbol{w}_{\boldsymbol{H} 2 \mathrm{O}}^{\text {DES }}$ [wt. \%] PC-SAFT $\boldsymbol{k}_{\boldsymbol{i j}}=0$ \\
\hline doca-lid 2:1 & - & 8.01 \\
\hline deca-lid 4:1 & $6.34 \pm 0.064$ & 2.38 \\
\hline deca-lid 3:1 & $8.25 \pm 0.65$ & 3.44 \\
\hline deca-lid 2:1 & $14.95 \pm 0.72$ & 3.89 \\
\hline deca-men & $2.16 \pm 0.01$ & 3.49 \\
\hline deca-thy & $3.42 \pm 0.01$ & 5.92 \\
\hline deca-n8888Br & $2.78 \pm 0.73$ & 4.25 \\
\hline thy-lid & $1.73 \pm 0.01$ & 3.93 \\
\hline
\end{tabular}

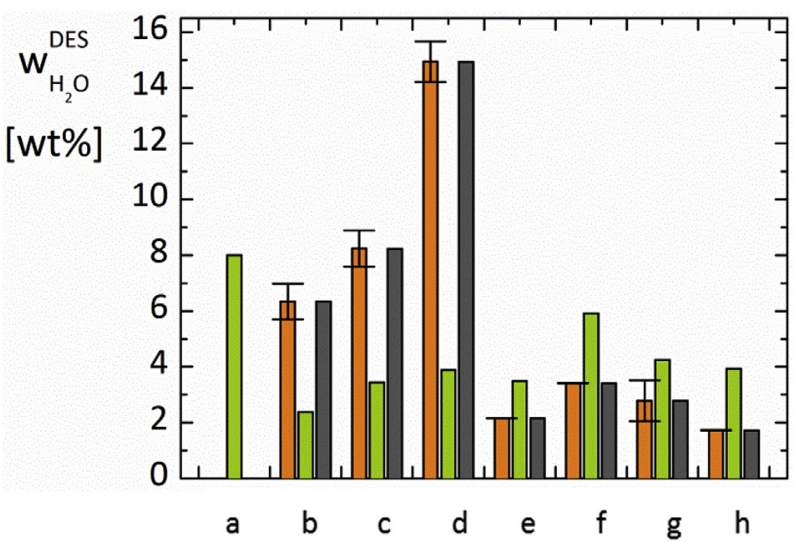

Fig. 3. Water weight fraction of the DES-rich phase of binary water + DES mixtures at $T=298 \mathrm{~K}$ and

$p=101 \mathrm{kPa}$. The binary mixtures are water + one of the following DESs: doca-lid (a), deca-lid 4:1 (b), deca:lid 3:1 (c), deca:lid 2:1 (d), deca-men (e), deca-thy (f), deca$\mathrm{n} 8888 \mathrm{Br}(\mathrm{g})$, and thy-lid $(\mathrm{h})$. Orange: experimental values from this work. Green PC-SAFT predictions using $k_{i j}=0$ between DES and water; grey: PC-SAFT modeling using the $k_{i j}$ values from Table 10 .

contrast, the unexpected experimental behavior of the systems water + deca-lid cannot be predicted quantitatively correct with PC-SAFT. It can be observed from Table 9 that PC-SAFT predicts water contents of about $3 \mathrm{wt} \%$ for the systems containing the DES deca-lid independent of the DESs composition. In contrast, the data show that more water will be present in the DES phase for systems with higher amounts of lidocaine over decanoic acid. This DES (deca-lid) might be seen in general as a very special system as not only this LLE data is unexpected, but already the vapor-pressure data ${ }^{[18]}$ of the DES showed strange behavior depending on the composition. The results on the LLE as illustrated in Fig. 3 might point to the fact that strong non-idealities between water and decalid DESs occur especially at high concentrations of lidocaine. At these conditions, PC-SAFT predictions using the pseudo-pure component modeling approach are not accurate enough. This can be overcome by using binary parameters or by applying the individual-constituent approach for modeling the DESs.

The latter was not within the focus of this work. Thus, $k_{i j}$ values were introduced between the DESs and water to more accurately model the water content of the DES phase. These were adjusted to the averaged experimental water contents of the DES phases, which are listed in Table 9. The resulting $k_{i j}$ values are listed in Table 10; the corresponding AARD\% values are given as well. Using these $k_{i j}$ values allows quantitatively modeling the water content in the DES phases especially for the systems containing the DES deca-lid, which was not possible quantitatively without the adjustable binary parameter.
Table 10

$k_{i j}$ values between water + DES and between HF + DES adjusted in this work.

\begin{tabular}{llll}
\hline Pair & $k_{i j}$ & fitted to & AARD\% [\%] \\
\hline Dode-lid/water & - & - & - \\
Deca-lid 4:1/water & -0.0633 & LLE (Table 9) & 0.03 \\
Deca-lid 3:1/water & -0.0518 & LLE (Table 9) & 0.12 \\
Deca-lid 2:1/water & -0.0645 & LLE (Table 9) & 0.10 \\
Deca-men/water & 0.0417 & LLE (Table 9) & 0.01 \\
Deca-thy/water & 0.028 & LLE (Table 9) & 0.18 \\
Deca-n8888Br/water & 0.0184 & LLE (Table 9) & 0.06 \\
Thy-lid/water & 0.0655 & LLE (Table 9) & 0.07 \\
Deca-thy/HMF & -0.0318 & SLE (Table 4) & 7.62 \\
Deca-n8888Br/HMF & -0.0485 & SLE (Table 4) & 4.08 \\
Thy-lid/HMF & 0.0065 & SLE (Table 4) & 0.55 \\
\hline
\end{tabular}

Finally, the HMF solubility in DESs was modeled. The results are illustrated in Fig. 4. PC-SAFT underpredicts the solubility of HMF in the considered DESs. Thus, $k_{i j}$ values (see Table 10) were introduced that allow quantitatively modeling of the HMF + DES mixtures.

In summary, Table 10 shows that PC-SAFT is a suitable model for DES-based mixtures with water or with sugar-derivatives such as HMF. Further, the pseudo-pure component modeling approach for the DESs seems to be sufficiently accurate to model phase behavior in good agreement to experimental data when using only one binary parameter (or in some cases even without any adjustable binary parameter). This is an important finding for future work on systems where sugar, water and a DES are commonly present; data on such systems are required for extraction (e.g. sugar from aqueous broths) or reaction purposes (e.g. sugar conversions in water or in DESs).

\section{Conclusions}

The density and total vapor pressure of eight different hydrophobic DESs were determined. Water solubility and HMF solubility in these DESs were measured. It was found that all hydrophobic DESs show low water solubility $\left(1.7 \mathrm{w} \%<w_{\text {water }}<14.4 \mathrm{w} \%\right)$ and high HMF solubility $\left(75 \mathrm{w} \%<w_{\mathrm{HMF}}<85 \mathrm{w} \%\right.$ ). Therefore, the hydrophobic DESs are interesting solvents for HMF removal from water in biorefinery processes. The obtained experimental data were validated by means of PC-SAFT modeling. New purecomponent parameters for the hydrophobic DES (considered as

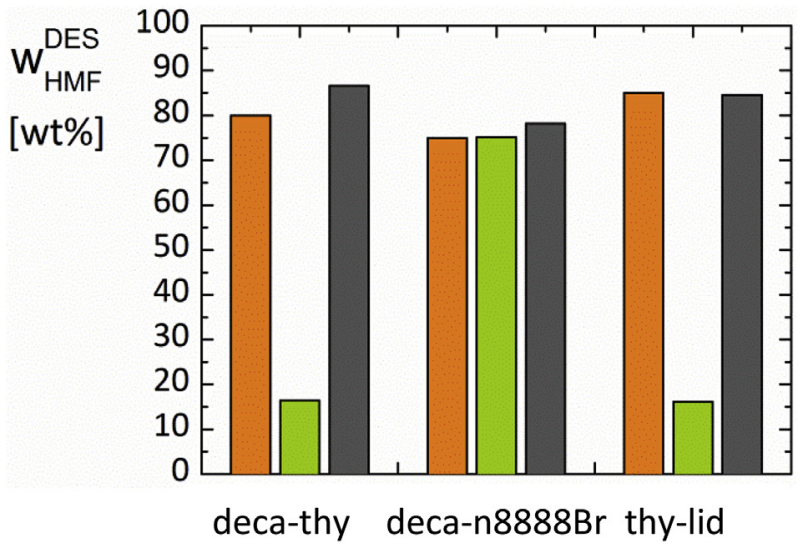

Fig. 4. Solubility of HMF in the DESs deca-thy, deca-n8888Br and thy-lid at $T=303 \mathrm{~K}$ and

$p=101 \mathrm{kPa}$. Orange: experimental values from this work; green: PC-SAFT predictions using $k_{i j}=0$ between DES and water; grey: PC-SAFT modeling using the $k_{i j}$ values from Table 10. 
pseudo-pure species) were obtained by fitting to both density and vapor-pressure data, instead of density data only. The density fittings were excellent, and the total vapor pressure fittings were reasonably well considering the low absolute vapor pressures. With the new PC-SAFT parameters, LLE of binary DES + water mixtures and SLE of binary DES + HMF mixtures were modeled using the pseudo-pure component modeling approach for the DESs. This modeling approach was found to be adequate for predicting the phase behavior of DES + water and DES + HMF systems, when using the binary parameter for the mixture dispersion-energy. This is important for the application of hydrophobic DESs in biorefineries, where HMF is often produced in aqueous solutions.

\section{Acknowledgements}

This work was performed under the framework of Chemelot InSciTe-Horizontal project and with contributions from the European Regional Development Fund (ERDF) within the framework of OP-Zuid and with contributions from the province of Brabant and Limburg and the Dutch Ministry of economic Affairs. Also, financial support from Max Buchner foundation is gratefully acknowledged.

\section{Appendix A. Supplementary data}

Supplementary data to this article can be found online at https://doi.org/10.1016/j.fluid.2019.02.010.

\section{References}

[1] P.T. Anastas, J.C. Warner, Green Chemistry: Theory and Practice, Oxford university press, 2000.

[2] P. Wasserscheid, T. Welton, Ionic Liquids in Synthesis, vol. 1, Wiley Online Library, Weinheim, 2008.

[3] A.P. Abbott, G. Capper, D.L. Davies, H.L. Munro, R.K. Rasheed, V. Tambyrajah, Chem. Commun. 0 (2001) 2010-2011.

[4] E.L. Smith, A.P. Abbott, K.S. Ryder, Chem. Rev. 114 (2014) 11060-11082.

[5] M. Francisco, A. van den Bruinhorst, M.C. Kroon, Angew. Chem. 52 (2013) 3074-3085.

[6] D.J.G.P. van Osch, L.F. Zubeir, A. van den Bruinhorst, M.A. Rocha, M.C. Kroon, Green Chem. 17 (2015) 4518-4521.

[7] B.D. Ribeiro, C. Florindo, L.C. Iff, M.A. Coelho, I.M. Marrucho, ACS Sustain.
Chem. Eng. 3 (2015) 2469-2477.

[8] D.J.G.P. van Osch, D. Parmentier, C.H.J.T. Dietz, A. van den Bruinhorst, R. Tuinier, M.C. Kroon, Chem. Commun. 52 (2016) 11987-11990.

[9] E. Tereshatov, M.Y. Boltoeva, C. Folden, Green Chem. 18 (2016) 4616-4622.

[10] C. Florindo, L. Branco, I. Marrucho, Fluid Phase Equilib. 448 (2017) 135-142.

[11] C.H. Dietz, M.C. Kroon, M. Di Stefano, M. van Sint Annaland, F. Gallucci, Faraday Discuss 206 (2017) 77-92.

[12] C.H.J.T. Dietz, D.J.G.P. van Osch, M.C. Kroon, G. Sadowski, M. van Sint Annaland, F. Gallucci, L.F. Zubeir, C. Held, Fluid Phase Equilib. 448 (2017) 94-98.

[13] M.B. Arain, E. Yilmaz, M. Soylak, J. Mol. Liq. 224 (2016) 538-543.

[14] S. Zhu, J. Zhou, H. Jia, H. Zhang, Food Chem. 243 (2018) 351-356.

[15] J. Cao, M. Yang, F. Cao, J. Wang, E. Su, ACS Sustain. Chem. Eng. 5 (2017) 3270-3278.

[16] E.L. de Faria, R.S. do Carmo, A.F.M. Cláudio, C.S. Freire, M.G. Freire, A.J. Silvestre, Int. J. Mol. Sci. 18 (2017) 2276.

[17] D.J.G.P. van Osch, C.H.J.T. Dietz, J. v. Spronsen, M.C. Kroon, F. Gallucci, M. v. Sint Annaland, Remco Tuinier, "A search for hydrophobic deep eutectic solvents based on natural components" submitted to, ACS Sustain. Chem. Eng. 7 (3) (2019) 2933-2942.

[18] C.H.J.T. Dietz, J.T. Creemers, M.A. Meuleman, C. Held, G. Sadowski, M. v. Sint Annaland, F. Gallucci, M.C. Kroon, "Determination of the Total Vapor Pressure of Hydrophobic Deep Eutectic Solvents: Experiments and PC-SAFT Modelling" Submitted to ACS Sustainable Chemistry \& Engineering, 2018, https://doi.org 10.1021/acssuschemeng.8b05449.

[19] B. Coto, R. van Grieken, J.L. Pena, J.J. Espada, Chem. Eng. Sci. 61 (2006) 8028-8039.

[20] J. Lecomte, A. Finiels, C. Moreau, Ind. Crops Prod. 9 (1999) 235-241.

[21] K.J. Zeitsch, The Chemistry and Technology of Furfural and its Many ByProducts, first ed., vol. 13, Sugar Series; Elsevier, The Netherlands, 2000.

[22] V. Giannetti, M.B. Mariani, P. Mannino, F. Marini, Volatile fraction analysis by HS-SPME/GC-MS and chemometric modeling for traceability of apples cultivated in the Northeast Italy, Food Control 78 (2017) 215 e221.

[23] M. Yamamoto, H. Narahara, The development of improved hydrogen chloride oxidation process, Submitimo Kagaku 1 (2004).

[24] J.M. Prausnitz, Molecular Thermodynamics of Fluid-phase Equilibria, PrenticeHall, Englewood Cliffs, N.J., 1969.

[25] J.G. Gmehling, T.F. Anderson, J.M. Prausnitz, Ind. Eng. Chem. Fundam. 17 (1978) 269-273.

[26] J. Gross, G. Sadowski, Ind. Eng. Chem. Res. 40 (2001) 1244-1260.

[27] J.P. Wolbach, S.I. Sandler, Ind. Eng. Chem. Res. 37 (1998) 2917-2928.

[28] L.F. Zubeir, C. Held, G. Sadowski, M.C. Kroon, J. Phys. Chem. B 120 (2016) $2300-2310$

[29] D. Fuchs, J. Fischer, F. Tumakaka, G. Sadowski, Ind. Eng. Chem. Res. 45 (2006 6578-6584.

[30] S. Mohammad, C. Held, E. Altuntepe, T. Köse, G. Sadowski, J. Phys. Chem. B 120 (2016) 3797-3808.

[31] S.P. Verevkin, V.N. Emel'yanenko, E.N. Ttepurko, R.V. Ralys, D.H. Zaitsau, A. Stark, Ind. Eng. Chem. Res. 48 (2009) 10087-10093. 